\title{
Global Sensitivity Analysis of Fuzzy Distribution Parameter on Failure Probability and Its Single-Loop Estimation
}

\author{
Lei Cheng, Zhenzhou Lu, and Luyi Li \\ School of Aeronautics, Northwestern Polytechnical University, P.O. Box 120, Xian, Shaanxi 710072, China \\ Correspondence should be addressed to Lei Cheng; mangoclei@mail.nwpu.edu.cn
}

Received 1 April 2014; Revised 24 June 2014; Accepted 24 June 2014; Published 15 July 2014

Academic Editor: Javier Oliver

Copyright (C) 2014 Lei Cheng et al. This is an open access article distributed under the Creative Commons Attribution License, which permits unrestricted use, distribution, and reproduction in any medium, provided the original work is properly cited.

\begin{abstract}
An extending Borgonovo's global sensitivity analysis is proposed to measure the influence of fuzzy distribution parameters on fuzzy failure probability by averaging the shift between the membership functions (MFs) of unconditional and conditional failure probability. The presented global sensitivity indices can reasonably reflect the influence of fuzzy-valued distribution parameters on the character of the failure probability, whereas solving the MFs of unconditional and conditional failure probability is timeconsuming due to the involved multiple-loop sampling and optimization operators. To overcome the large computational cost, a single-loop simulation (SLS) is introduced to estimate the global sensitivity indices. By establishing a sampling probability density, only a set of samples of input variables are essential to evaluate the MFs of unconditional and conditional failure probability in the presented SLS method. Significance of the global sensitivity indices can be verified and demonstrated through several numerical and engineering examples.
\end{abstract}

\section{Introduction}

Two different uncertainty sources are involved in reliability engineering: aleatory uncertainty and epistemic uncertainty [1-3]. Aleatory uncertainty describes the inherent variability associated with a structural system, which is referred to as irreducible, objective uncertainty, while epistemic uncertainty results from lack of knowledge of fundamental phenomena and is related to our ability to understand, measure, and describe the system under study. Employing the probability theory, the aleatory uncertainty can be modeled as random variable with probability density function (PDF). When dealing with the epistemic uncertainty, more than one theory has been developed, such as the interval analysis [4$6]$, the evidence theory $[7,8]$, and the fuzzy set theory [9].

Input variables can be modeled as random variables when pure aleatory uncertainty is present. Thus available sensitivity analysis (SA) techniques can be applied to study how uncertainty in the output of a model can be apportioned to different sources of uncertainty in the model input. SA is commonly divided into two groups: local SA (LSA) and global SA (GSA). LSA is defined as the partial derivatives of model output with respect to model input, which is generally computed at one given point. GSA, also known as importance analysis, focuses on determining which of the input variables affects output most in the whole uncertainty. With the ranking of the input variables resulting from importance analysis, one can pay more attention to variables with high importance and neglect variables with low importance during the process of design and optimization, thus providing useful information and guidance for engineers. At present, many GSA techniques and indices are available in the case of randomness, such as nonparametric techniques [10-12], variance-based importance measure indices [13-16], and moment-independent importance measures [17-19], among which moment independent sensitivity analysis is attracting growing attention among both academics and practitioners. The moment independent sensitivity index $\delta_{i}$ proposed by Borgonovo is widely extended in GSA: Castaings et al. [20], Plischke et al. [21], and Luo et al. [22] developed methods for moment independent indices recently; the moment independent model was applied to environmental models [23] and climate model [24] et al.; Wei et al. [25] and Borgonovo et al. [26] brought up new moment independent indices.

When fuzzy set theory is employed, assigned membership function (MF) is used to model the epistemic uncertainty. 
Subsequently, methods for calculating the possibility distribution of output response are proposed [27-32]. Based on the fuzzy set theory which models the epistemic uncertainty as a fuzzy variable represented by MF, some methods have been developed for evaluating the importance of fuzzy variables [29-31, 33], among which Song et al. [33] proposed a generalized Borgonovo's sensitivity indicator to analyze the effect of fuzzy-valued variables on the output response, and optimization techniques are employed to calculate the MF of output response [34]. When two kinds of uncertainties are concomitant in reliability problems, where aleatory uncertainty is described by PDF and epistemic uncertainty is modeled by fuzzy set theory, GSA for both fuzzy and random input variables is constructed by Zhangchun et al. in recent years [35-37].

However, obviously, the importance of basic variable is affected by distribution parameters of all involved basic random variables which need to be designed for the required output response. Therefore, it is essential to analyze the importance of distribution parameter on probabilistic response in engineering applications. Recently, GSA is constructed for systems with both input variables and distribution parameters described by PDF [38-40], whereas in this paper, the input variables are described by PDF and the distribution parameters are modeled as fuzzy parameters with assigned MF [41-43].

Then, a global sensitivity analysis is proposed to estimate the effect of fuzzy-valued distribution parameters on failure probability. Estimation of the established global sensitivity indices involves calculating the MFs of unconditional and conditional failure probability. For the problem that the computational cost for direct Monte-Carlo simulation (MCS) is rather too expensive to be accepted, a single-loop simulation (SLS) based on the concept of importance sampling is introduced to calculate the failure probability function (FPF), where a sampling PDF is introduced to generate a group of samples of the input variables. Thus, only one set of function calls is necessary to evaluate the sensitivity indices, and the efficiency has been greatly improved comparing with the existed direct MCS.

The paper is organized as follows. In Section 2, sensitivity indices of fuzzy-valued distribution parameters on failure probability are proposed and their properties are established. Section 3 gives the detailed procedures for direct MCS. Section 4 presents the SLS which can greatly save the computation cost. Applicability of sensitivity indices and accuracy and efficiency of the SLS are demonstrated by the numerical and engineering examples in Section 5. The paper ends with conclusions in Section 6.

\section{Global Sensitivity Indices for Fuzzy Distribution Parameter Based on Failure Probability}

2.1. Definitions of Global Sensitivity Indices. Considering structural systems with both epistemic and aleatory uncertainties, the output response can be given as

$$
Y=g(\mathbf{X}),
$$

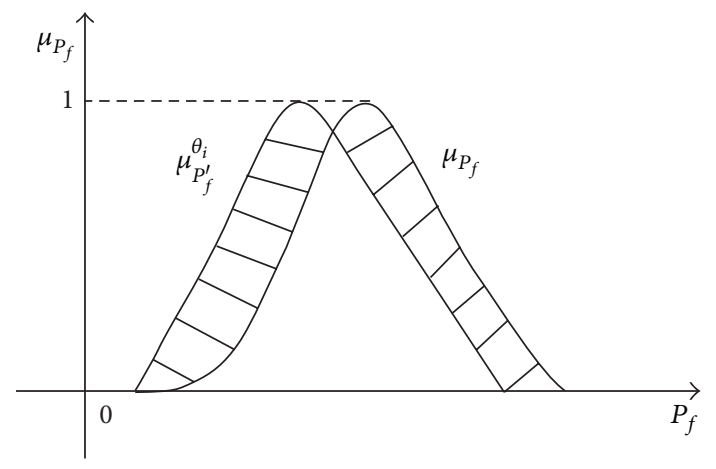

FIGURE 1: Shift between $\mu_{P_{f}}$ and $\mu_{P_{f}^{\prime}}^{\theta_{i}}$.

where $Y$ is the output response. Let $\mathbf{X}$ denote the aleatory variables whose PDF is given by $f_{\mathbf{X}}\left(\mathbf{x} \mid \boldsymbol{\theta}^{*}\right)$, and $\boldsymbol{\theta}=$ $\left(\theta_{1}, \theta_{2}, \ldots, \theta_{p}\right)$ refers to the fuzzy-valued distribution parameter vector, and its characteristic is represented by a family of MFs noted by $\mu_{\theta_{i}}\left(\theta_{i}\right)(i=1,2, \ldots, p)$. The membership grade $\mu_{\theta_{i}}(\omega)$ quantifies the degree of possibility that parameter $\theta_{i}$ takes value $\omega$. It is possible to evaluate fuzzy parameters using $\lambda$-cuts or membership levels. Generally, the cut level $\lambda$ takes value from the interval $[0,1]$. At each $\lambda$-cut level, the variation of the fuzzy variable is defined by a lower bound $\theta_{i L}(\lambda)$ and upper bound $\theta_{i U}(\lambda)$.

Once the bounds of distribution parameters at $\lambda$-cut are obtained by the corresponding MF $\mu_{\theta_{i}}\left(\theta_{i}\right)$, the bounds $\left[P_{f}^{L}(\boldsymbol{\theta}(\lambda)), P_{f}^{U}(\boldsymbol{\theta}(\lambda))\right]$ of failure probability at various $\lambda$-cut can be calculated; that is, the MF $\mu_{P_{f}}$ of unconditional failure probability is obtained.

While the $i$ th distribution parameter $\theta_{i}$ was fixed at a value $\theta_{i}^{*}$, that is, $\boldsymbol{\theta}^{*}=\left(\theta_{1}, \ldots, \theta_{i-1}, \theta_{i}^{*}, \theta_{i+1}, \ldots, \theta_{p}\right)$, the MF $\mu_{P_{f}^{\prime}}^{\theta_{i}}$ of conditional failure probability can also be evaluated. Obviously, the shift between $\mu_{P_{f}}$ and $\mu_{P_{f}^{\prime}}^{\theta_{i}}$ measured by the area $s_{\theta_{i}}$, which is the shadow area shown in Figure 1, can reflect the effect of the fixed parameter $\theta_{i}$ on failure probability:

$$
s_{\theta_{i}}=\int_{0}^{1}\left|\mu_{P_{f}}-\mu_{P_{f}^{\prime}}^{\theta_{i}}\right| d P_{f} .
$$

The larger the value of $s_{\theta_{i}}$ is, the more important the fixed value $\theta_{i}$ for failure probability is. In average, the global sensitivity index of $\theta_{i}$ can be measured by

$$
\tau_{\theta_{i}}=\int_{-\infty}^{+\infty} \mu_{\theta_{i}} s_{\theta_{i}} d \theta_{i} .
$$

However, in the engineering, the fuzzy-valued distribution parameters may have different dimensions. In order to eliminate the infections of the units of distribution parameters, we normalize the distribution parameters by introducing the equivalent $\operatorname{PDF} \mu_{\theta_{i}}^{(e)}=\mu_{\theta_{i}} / \int \mu_{\theta_{i}} d \theta_{i}$, which satisfies the property that $\int \mu_{\theta_{i}}^{(e)} d \theta_{i}=1$. The equivalent PDF can eliminate the effect of the dimensions, while it does not change the 
distribution type of the MFs of distribution parameters [44] Thus, we have

$$
\begin{aligned}
\Gamma_{\theta_{i}} & =\int_{-\infty}^{+\infty} \frac{\mu_{\theta_{i}}}{\int \mu_{\theta_{i}} d \theta_{i}} s_{\theta_{i}} d \theta_{i} \\
& =\int_{-\infty}^{+\infty} \frac{\mu_{\theta_{i}}}{\int \mu_{\theta_{i}} d \theta_{i}} \int_{0}^{1}\left|\mu_{P_{f}}-\mu_{P_{f}^{\prime}}^{\theta_{i}}\right| d P_{f} d \theta_{i} .
\end{aligned}
$$

From the above definitions, we know that $\Gamma_{\theta_{i}}$ represents the effect of the distribution parameter $\theta_{i}$ on failure probability of structural model.

Generally, MFs of failure probability cannot be expressed by explicit expression, which are determined by the lower and upper bounds at various $\lambda$-cuts. So the integral of (4) needs numerical integration to be evaluated; that is,

$$
\begin{aligned}
\Gamma_{\theta_{i}}=\sum_{j=1}^{N}\{ & \Delta \theta_{i} \cdot \frac{\mu_{\theta_{i}}\left(\theta_{i, j}\right)}{\int \mu_{\theta_{i}} d \theta_{i}} \\
& \left.\times \sum_{k=1}^{M}\left[\Delta P_{f} \cdot\left|\mu_{P_{f}}\left(P_{f}^{(k)}\right)-\mu_{P_{f}^{\prime}}^{\theta_{i}}\left(P_{f}^{(k)}\right)\right|\right]\right\},
\end{aligned}
$$

where $\Delta \theta_{i}$ and $\Delta P_{f}$ are the spans of isometric sampling, $\theta_{i, j}$ is the $j$ th fixed value of the distribution parameter $\theta_{i}$, and $P_{f}^{(k)}$ is the $k$ th value of unconditional failure probability $P_{f}$.

Global sensitivity index for an individual distribution parameter can be easily extended to a group of distribution parameters. Then the sensitivity index for a group of parameters can be given as:

$$
\begin{aligned}
\Gamma_{\theta_{i}, \theta_{j}}=\int_{-\infty}^{+\infty} \int_{-\infty}^{+\infty} \frac{\mu_{\theta_{i}}}{\int \mu_{\theta_{i}} d \theta_{i}} \frac{\mu_{\theta_{j}}}{\int \mu_{\theta_{j}} d \theta_{j}} \\
\quad \times \int_{0}^{1}\left|\mu_{P_{f}}-\mu_{P_{f}^{\prime}}^{\theta_{i}, \theta_{j}}\right| d P_{f} d \theta_{i} d \theta_{j} \\
\Gamma_{\theta_{i} \mid \theta_{j}}=\int_{-\infty}^{+\infty} \int_{-\infty}^{+\infty} \frac{\mu_{\theta_{i}}}{\int \mu_{\theta_{i}} d \theta_{i}} \frac{\mu_{\theta_{j}}}{\int \mu_{\theta_{j}} d \theta_{j}} \\
\times \int_{0}^{1}\left|\mu_{P_{f}^{\prime}}^{\theta_{i}}-\mu_{P_{f}^{\prime}}^{\theta_{i}, \theta_{j}}\right| d P_{f} d \theta_{i} d \theta_{j},
\end{aligned}
$$

where $\mu_{P_{f}^{\prime}}^{\theta_{i}, \theta_{j}}$ is the MF of conditional failure probability with distribution parameters $\theta_{i}$ and $\theta_{j}$ taken the fixed values $\theta_{i}^{*}$ and $\theta_{j}^{*}$, respectively.
Similarly, the numerical integrations for (6) are shown as follows:

$$
\begin{aligned}
\Gamma_{\theta_{i}, \theta_{j}}=\sum_{l=1}^{N_{i}} \sum_{m=1}^{N_{j}} \Delta \theta_{i} \Delta \theta_{j} \cdot \frac{\mu_{\theta_{i}}\left(\theta_{i, l}\right)}{\int \mu_{\theta_{i}} d \theta_{i}} \frac{\mu_{\theta_{j}}\left(\theta_{j, m}\right)}{\int \mu_{\theta_{j}} d \theta_{j}} \\
\times\left\{\sum_{q=1}^{M}\left[\Delta P_{f} \cdot\left|\mu_{P_{f}}\left(P_{f}^{(k)}\right)-\mu_{P_{f}^{\prime}}^{\theta_{i}, \theta_{j}}\left(P_{f}^{(k)}\right)\right|\right]\right\} \\
\Gamma_{\theta_{i} \mid \theta_{j}}=\sum_{l=1}^{N_{i}} \sum_{m=1}^{N_{j}} \Delta \theta_{i} \Delta \theta_{j} \cdot \frac{\mu_{\theta_{i}}\left(\theta_{i, l}\right)}{\int \mu_{\theta_{i}} d \theta_{i}} \frac{\mu_{\theta_{j}}\left(\theta_{j, m}\right)}{\int \mu_{\theta_{j}} d \theta_{j}} \\
\times\left\{\sum_{q=1}^{M}\left[\Delta P_{f} \cdot\left|\mu_{P_{f}^{\prime}}^{\theta_{i}}\left(P_{f}^{(k)}\right)-\mu_{P_{f}^{\prime}}^{\theta_{i}, \theta_{j}}\left(P_{f}^{(k)}\right)\right|\right]\right\} .
\end{aligned}
$$

Mathematical properties of the sensitivity indices are discussed in Section 2.2.

2.2. Mathematical Properties of the Proposed Sensitivity Indices. Similar to the sensitivity indices of the random input variables proposed by Song et al. [33], the mathematical properties of the proposed sensitivity indices for fuzzy distribution parameters can be explained as follows.

Property 1. $\Gamma_{\theta_{i}} \geq 0$.

Property 2. If failure probability $P_{f}$ is independent of $\theta_{i}$, then $\Gamma_{\theta_{i}}=0$.

Property 3. If $P_{f}$ is dependent of $\theta_{i}$ but independent of $\theta_{j}$, then $\Gamma_{\theta_{i} \theta_{j}}=\Gamma_{\theta_{i}}, \Gamma_{\theta_{i} \mid \theta_{j}}=0$, and $\Gamma_{\theta_{j} \mid \theta_{i}}=\Gamma_{\theta_{i}}$.

Proofs for the above properties are similar with those in [19].

Note that since the sensitivity index $\delta_{i}$ proposed by Borgonovo et al. has the property of transformation invariance $[45,46]$, thus the algorithms of computing the delta index can converge even faster if a monotonic transformation is applied when dealing with large distribution region of the model output. From the definitions in (2) and (3), we can see that the inner statistic of $\Gamma_{\theta_{i}}$ and inner statistic of $\delta_{i}$ both are $L^{1}$ norm on densities; thus; the moment independent sensitivity index for fuzzy-valued parameters is also monotonic transformation invariant. For knowing about the detailed property of moment-independent sensitivity index, one could refer to $[45,46]$.

\section{MCS for Evaluating MFs of Failure Probability}

Failure probability $P_{f}$ is a function of the basic fuzzy-valued distribution parameter $\boldsymbol{\theta}=\left(\theta_{1}, \theta_{2}, \ldots, \theta_{p}\right)$, given by $P_{f}=$ $\psi\left(\theta_{1}, \theta_{2}, \ldots, \theta_{p}\right)$. Details of MCS for evaluating the MF of failure probability are as follows.

(1) At $\lambda_{k}$-cut $\left(k=1,2, \ldots, N_{\lambda}\right)$, the lower bounds and upper bounds of the parameters $\boldsymbol{\theta}=\left(\theta_{1}, \theta_{2}, \ldots, \theta_{p}\right)$ 
can be obtained as $\left[\theta_{i L}\left(\lambda_{k}\right), \theta_{i U}\left(\lambda_{k}\right)\right](i=1,2, \ldots, p)$ according to the MF $\mu_{\theta_{i}}\left(\theta_{i}\right)$ of basic fuzzy-valued parameter $\theta_{i}$. Generate $N$ samples $\boldsymbol{\theta}_{t}=\left(\theta_{1 t}\right.$, $\left.\theta_{2 t}, \ldots, \theta_{p t}\right)(t=1,2, \ldots, N)$ according to the intervals of distribution parameters at the given $\lambda_{k}$-cut.

(2) For each sample $\boldsymbol{\theta}_{t}$, generate $N$ samples $\mathbf{x}_{s}=\left(x_{1 s}\right.$, $\left.x_{2 s}, \ldots, x_{n s}\right)(s=1,2, \ldots, N)$ of aleatory variables $\mathbf{X}$, and then the corresponding $N$ samples $P_{f}^{s}(s=$ $1,2, \ldots, N)$ of failure probability $P_{f}$ can be evaluated. Thus, the lower bound $P_{f}^{L}\left(\lambda_{k}\right)$ and upper bound $P_{f}^{U}\left(\lambda_{k}\right)$ of unconditional failure probability at given $\lambda_{k}$-cut can be obtained.

(3) Iterate (1) and (2) for $N_{\lambda}$ times; then, the MF of unconditional failure probability $\mu_{P_{f}}$ is evaluated.

(4) Change parameter matrix $\boldsymbol{\theta}_{t}=\left(\theta_{1 t}, \theta_{2 t}, \ldots, \theta_{p t}\right)$ to $\boldsymbol{\theta}_{t}^{*}=\left(\theta_{1 t}, \theta_{2 t}, \ldots, \theta_{i t}^{*}, \ldots, \theta_{p t}\right)$ by fixing the $i$ th distribution parameter $\theta_{i}$ at its fixed value $\theta_{i t}(i=$ $1,2, \ldots, p, t=1,2, \ldots, N)$.

(5) For each sample $\boldsymbol{\theta}_{t}^{*}$, generate $N$ samples $\mathbf{x}_{s}^{*}=\left(x_{1 s}\right.$, $\left.x_{2 s}, \ldots, x_{n s}\right)(s=1,2, \ldots, N)$ of aleatory variables $\mathbf{X}$; then, the corresponding $N$ samples $P_{f}^{s^{*}}(s=$ $1,2, \ldots, M)$ of failure probability $P_{f}^{*}$ can be evaluated. Thus, the lower bound $P_{f}^{L^{*}}\left(\lambda_{k}\right)$ and upper bound $P_{f}^{U^{*}}\left(\lambda_{k}\right)$ of conditional failure probability at given $\lambda_{k}{ }^{-}$ cut can be obtained.

(6) Iterate (4) and (5) for $N$ times; then, the $N$ MFs $\mu_{P_{f} \mid \theta_{i}=\theta_{i t}}(t=1,2, \ldots, N)$ of conditional failure probability can be obtained.

(7) Iterate (4), (5), and (6) for $p$ times; all the MFs of conditional failure probability of distribution parameters $\mu_{P_{f} \mid \theta_{j}=\theta_{j t}}(j=1,2, \ldots, p, t=1,2, \ldots, N)$ can be evaluated.

We can see from the procedures in Figure 2 that using MCS combing optimization techniques $N \times N \times N_{\lambda}$ and $p \times N \times N \times N \times N_{\lambda}$ function calls are needed to calculate the MFs of unconditional and conditional failure probability, which is unacceptable especially in engineering problems. Thus, a single-loop method is proposed to evaluate the FPF of the distribution parameters in Section 4. ( $N$ is the sample number of distribution parameters and $N_{\lambda}$ is the cut number of the membership functions.)

\section{SLS for Evaluating MFs of Failure Probability}

By definition, FPF is expressed as

$$
P_{f}(\boldsymbol{\theta})=\int_{g(\mathbf{x}) \leq 0} I_{F}(\mathbf{x}) f(\mathbf{x} \boldsymbol{\theta}) d \mathbf{x}
$$

Suppose now we have a joint PDF $h_{\mathbf{x}}(\mathbf{x})$ of model input vector with constant distribution parameter vector $\boldsymbol{\theta}^{*}=$ $\left(\theta_{1}^{*}, \theta_{2}^{*}, \ldots, \theta_{m}^{*}\right)$. Then the FPF can be derived as

$$
\begin{aligned}
P_{f}(\boldsymbol{\theta}) & =\int \frac{I_{F}(\mathbf{x}) f_{\mathbf{x}}(\mathbf{x} \mid \boldsymbol{\theta})}{h_{\mathbf{x}}(\mathbf{x})} h_{\mathbf{x}}(\mathbf{x}) d \mathbf{x} \\
& =E_{h}\left(\frac{I_{F}(\mathbf{x}) f_{\mathbf{x}}(\mathbf{x} \mid \boldsymbol{\theta})}{h_{\mathbf{x}}(\mathbf{x})}\right),
\end{aligned}
$$

where $h_{\mathbf{x}}(\mathbf{x})$ is an arbitrary selected joint PDF of $\mathbf{X}$ with constant distribution parameters $\boldsymbol{\theta}^{*}$. Equation (9) indicates that $P_{f}(\boldsymbol{\theta})$ can be expressed as the expectation of the function $I_{F}(\mathbf{x}) f_{\mathbf{x}}(\mathbf{x} \mid \boldsymbol{\theta}) / h_{\mathbf{x}}(\mathbf{x})$. Given a set of model input vector samples $\mathbf{x}^{(k)}=\left(x_{1}^{(k)}, x_{2}^{(k)}, \ldots, x_{n}^{(k)}\right)(k=1,2, \ldots, N)$ generated by the joint PDF $h_{\mathbf{x}}(\mathbf{x})$, then $P_{f}(\boldsymbol{\theta})$ can be estimated by

$$
\widehat{P}_{f}(\boldsymbol{\theta})=\frac{1}{N} \sum_{k=1}^{N} \frac{I_{F}\left(\mathbf{x}^{(k)}\right) f_{\mathbf{x}}\left(\mathbf{x}^{(k)} \boldsymbol{\theta}\right)}{h_{\mathbf{x}}\left(\mathbf{x}^{(k)}\right)} .
$$

Detailed procedures for SLS are as follows.

(1) Generate $N$ samples $\mathbf{x}_{s}=\left(x_{1 s}, x_{2 s}, \ldots, x_{n s}\right)(s=$ $1,2, \ldots, N)$ according to the joint PDF $h_{\mathbf{x}}(\mathbf{x})$. For each $\mathbf{x}_{s}(s=1,2, \ldots, N)$, calculate the values of selected joint $\operatorname{PDF} h_{\mathbf{x}}\left(\mathbf{x}_{s}\right)$ and failure indicator $I_{F}\left(\mathbf{x}_{s}\right)$ as $h^{s}$ and $I_{F}^{s}$.

(2) At $\lambda_{k}$-cut $\left(k=1,2, \ldots, N_{\lambda}\right)$, the lower and upper bounds of the parameters $\boldsymbol{\theta}=\left(\theta_{1}, \theta_{2}, \ldots, \theta_{p}\right)$ can be obtained as $\left[\theta_{i L}\left(\lambda_{k}\right), \theta_{i U}\left(\lambda_{k}\right)\right](i=1,2, \ldots, p)$ according to the MF $\mu_{\theta_{i}}\left(\theta_{i}\right)$ of basic fuzzy-valued parameter. Generate $N$ samples $\boldsymbol{\theta}_{t}=\left(\theta_{1 t}, \theta_{2 t}, \ldots, \theta_{p t}\right)(t=$ $1,2, \ldots, N)$ according to the intervals of distribution parameters at the given $\lambda_{k}$-cut.

(3) For each $\boldsymbol{\theta}_{t}(t=1,2, \ldots, N)$, calculate the values of the original joint PDF $f_{\mathbf{x}}\left(\mathbf{x}_{s} \mid \boldsymbol{\theta}\right)$ as $f^{s}$. Then (10) can be calculated as $(1 / N) \sum_{s=1}^{N} I_{F}^{s} f^{s} / h^{s}$; that is, $\widehat{P}_{f}\left(\boldsymbol{\theta}_{t}\right)$ is obtained.

(4) Iterate (3) for $N$ times; then, $\widehat{P}_{f}(\boldsymbol{\theta})$ is calculated. Thus, the lower bound $\widehat{P}_{f}^{L}$ and upper bound $\widehat{P}_{f}^{U}$ of unconditional failure probability at given $\lambda_{k}$-cut are obtained.

(5) Iterate (3) and (4) for $N_{\lambda}$ times, and the MF $\mu_{P_{f}}$ of unconditional failure probability can be evaluated.

(6) Change parameter matrix $\boldsymbol{\theta}_{t}=\left(\theta_{1 t}, \theta_{2 t}, \ldots, \theta_{p t}\right)$ to $\boldsymbol{\theta}_{t}^{*}=\left(\theta_{1 t}, \theta_{2 t}, \ldots, \theta_{i t}^{*}, \ldots, \theta_{p t}\right)$ by fixing the $i$ th distribution parameter $\theta_{i}$ at its fixed value $\theta_{i t}(i=$ $1,2, \ldots, p, t=1,2, \ldots, N)$.

(7) For each $\boldsymbol{\theta}_{t}^{*}(t=1,2, \ldots, N)$, calculate the values of the original joint $\operatorname{PDF} f_{\mathbf{x}}\left(\mathbf{x}_{s} \mid \boldsymbol{\theta}\right)$ as $f^{s^{*}}$. Then (10) can be calculated as $(1 / N) \sum_{k=1}^{N} I_{F}^{s} f^{s^{*}} / h^{s}$; that is, $\widehat{P}_{f}\left(\boldsymbol{\theta}_{t}^{*}\right)$ is obtained.

(8) Iterate (7) for $N$ times; then, $\widehat{P}_{f}\left(\boldsymbol{\theta}^{*}\right)$ is calculated. Thus, the lower bound $\widehat{P}_{f}^{L^{*}}$ and upper bound $\widehat{P}_{f}^{U^{*}}$ of conditional failure probability at given $\lambda_{k}$-cut are obtained. 


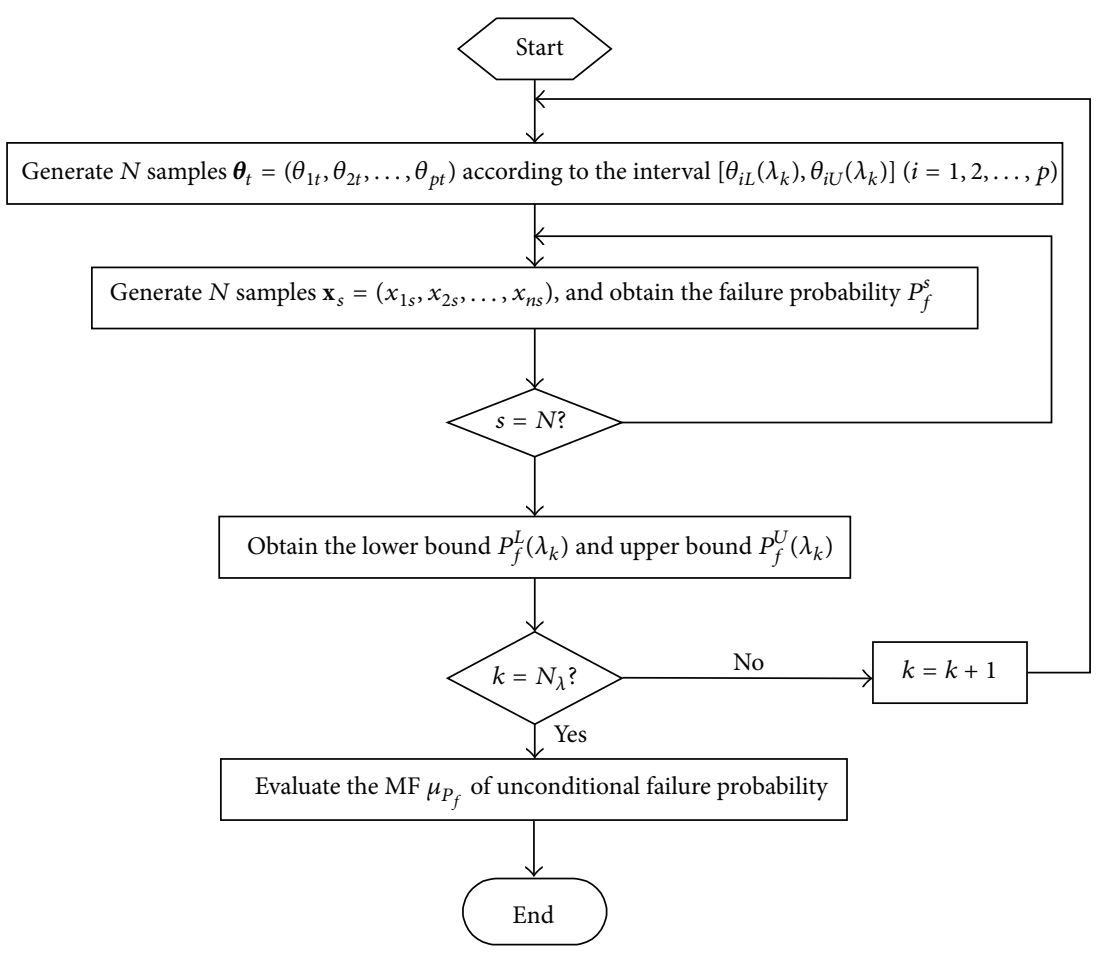

(a) MCS for evaluating MF of unconditional failure probability

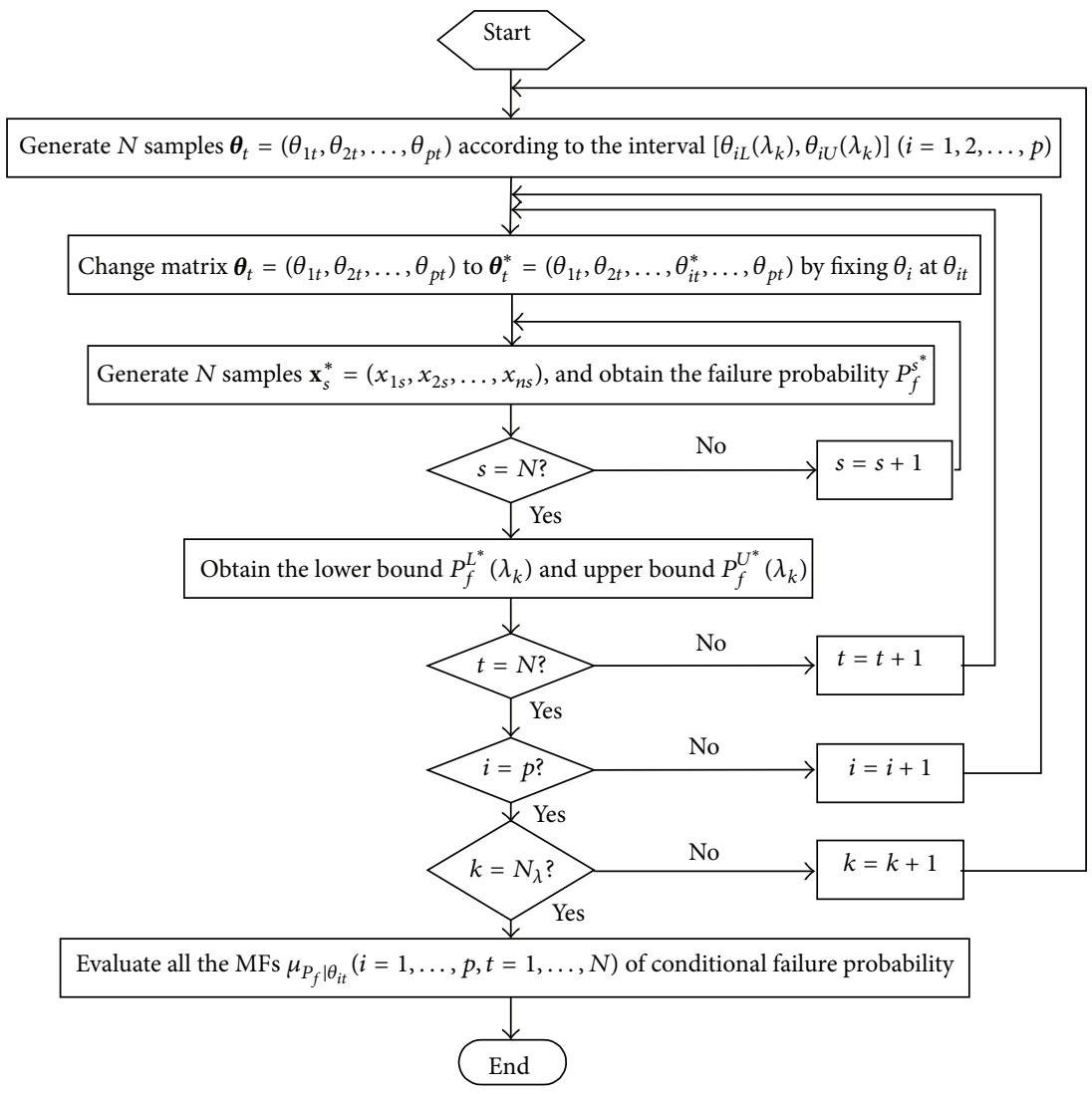

(b) MCS for evaluating MFs of conditional failure probability

FIGURE 2: Flow chart of MCS for evaluating the MFs of unconditional and conditional failure probability. 
TABLE 1: Results of global sensitivity indices for Example 1, Case 1.

\begin{tabular}{lcc}
\hline & MCS & SLS \\
\hline$\left\{\Gamma_{\mu_{1}}, \Gamma_{\mu_{2}}\right\}$ & $\{0.0461,0.0461\}$ & $\{0.0458,0.0463\}$ \\
$\left\{\Gamma_{\mu_{1} \mu_{2}}\right\}$ & $\{0.0356\}$ & $\{0.0362\}$ \\
$\left\{\Gamma_{\mu_{1} \mid \mu_{2}}, \Gamma_{\mu_{2} \mid \mu_{1}}\right\}$ & $\{0.0192,0.0193\}$ & $\{0.0187,0.0189\}$ \\
Function Calls & $p \times N \times N \times N \times N_{\lambda}+N \times N \times N_{\lambda}$ & $N$ \\
$\left(N=2000, N_{\lambda}=21, p=2\right)$ & & \\
\hline
\end{tabular}

(9) Iterate (7) and (8) for $N_{\lambda}$ times, and the MF $\mu_{P_{f} \mid \theta_{i}=\theta_{i t}}$ of conditional failure probability can be evaluated.

(10) Iterate (7), (8), and (9) for $N \times p$ times, and all the MFs $\mu_{P_{f} \mid \theta_{i}=\theta_{i t}}(i=1,2, \ldots, p, t=1,2, \ldots, N)$ of conditional failure probability are evaluated.

The procedure in Figure 3 shows that given any $\lambda_{k}$-cut $\left(k=1,2, \ldots, N_{\lambda}\right)$, the interval of distribution parameters $\left[\boldsymbol{\theta}_{L}\left(\lambda_{k}\right), \boldsymbol{\theta}_{U}\left(\lambda_{k}\right)\right]$ can be obtained; thus, the failure probability can be estimated using (10); that is, (10) can be treated as the imitate explicit function of the distribution parameters. Then any arbitrary optimization method can be used to get the upper and lower bounds of failure probability $\left[P_{f}^{L}\left(\lambda_{k}\right), P_{f}^{U}\left(\lambda_{k}\right)\right]$ at given interval $\left[\boldsymbol{\theta}_{L}\left(\lambda_{k}\right), \boldsymbol{\theta}_{U}\left(\lambda_{k}\right)\right]$ of distribution parameters. Only one set of samples of the aleatory variables is used in the procedure at different $\lambda_{k}$ cut and only $N$ function calls are needed to calculate the MF of unconditional failure probability. The procedures for calculating the MFs of conditional failure probability are similar, and the only difference is that the one or a group of distribution parameters are fixed at certain value.

Hence the above procedures for estimating the FPF need only one set of samples for implementing it; that is, the function calls for SLS is $N$; thus, it is computationally efficient.

Considering that the computational cost is rather expensive, optimization techniques can be employed to calculate the minimum and maximum values of the failure probability within the specified bounds of the cuts of the fuzzy-valued parameters corresponding to $\lambda$-cut. Thus, combing MCS and SLS with local/global optimization techniques can be efficient. Accuracy and efficiency of the SLS can be established in Section 5.

\section{Examples}

A number of examples are used to demonstrate the availability and feasibility of the presented sensitivity indicator. These examples involve some cases met in engineering commonly, for example, normal, triangular, or trapezoidal types MFs, and MFs of failure probability are calculated by employing the optimization techniques.

Example 1. The response function $z=4-x_{1}-x_{2}$ has two variables. Case 1: $x_{i} \sim N\left(\mu_{i}, 2\right), \mu_{i}(i=1,2)$ are fuzzy-valued
TABLE 2: Results of global sensitivity indices for Example 1, Case 2.

\begin{tabular}{lcc}
\hline & MCS & SLS \\
\hline$\left\{\Gamma_{\sigma_{1}}, \Gamma_{\sigma_{2}}\right\}$ & $\{0.0172,0.0178\}$ & $\{0.0175,0.0173\}$ \\
$\left\{\Gamma_{\sigma_{1} \sigma_{2}}\right\}$ & $\{0.0281\}$ & $\{0.0279\}$ \\
$\left\{\Gamma_{\sigma_{1} \mid \sigma_{2}}, \Gamma_{\sigma_{2} \mid \sigma_{1}}\right\}$ & $\{0.0149,0.0147\}$ & $\{0.0151,0.0146\}$ \\
\hline
\end{tabular}

distribution parameters. Case 2: $x_{i} \sim N\left(1, \sigma_{i}\right), \sigma_{i}(i=1,2)$ are fuzzy-valued parameters. The MFs are listed as follows:

$$
\begin{aligned}
& \mu_{\mu_{i}}\left(\mu_{i}\right)= \begin{cases}4-\mu_{i} & 3 \leq \mu_{i} \leq 4 \\
\mu_{i}-2 & 2 \leq \mu_{i} \leq 3,\end{cases} \\
& \mu_{\sigma_{i}}\left(\sigma_{i}\right)= \begin{cases}4-\sigma_{i} & 3 \leq \sigma_{i} \leq 4 \\
\sigma_{i}-2 & 2 \leq \sigma_{i} \leq 3 .\end{cases}
\end{aligned}
$$

The MFs of failure probability are drawn in Figures 4 and 5, and the results of global sensitivity indices calculated by MCS and SLS combing with optimization techniques are listed in Table 1.

\section{Case 1. See Figure 4 and Table 1.}

Case 2. See Figure 5 and Table 2.

Figures 4 and 5 show that the MFs of failure probability obtained by the SLS are in good accordance with those obtained by MCS, which testifies the accuracy of SLS. And Tables 1 and 2 indicate that the sensitivity indices of the two distribution parameters are equal, which is reasonable in this example. In addition, the accuracy of SLS is proved again by the results estimated by the two methods in Tables 1 and 2. In addition, the function calls of the two methods are also listed in Table 1, proving that SLS is greatly efficient in evaluating the proposed sensitivity indices.

To make the numerical results more robust, we carry out the calculations of sensitivity indices at increasing sample sizes to demonstrate convergence. Figures 6 and 7 show the convergence plot for Case 1 using MCS and SLS, respectively. One can easily find that the results begin to converge at the level of $2 \times 10^{3}$. Thus we take $N=2 \times 10^{3}$ as shown in Table 1 . 


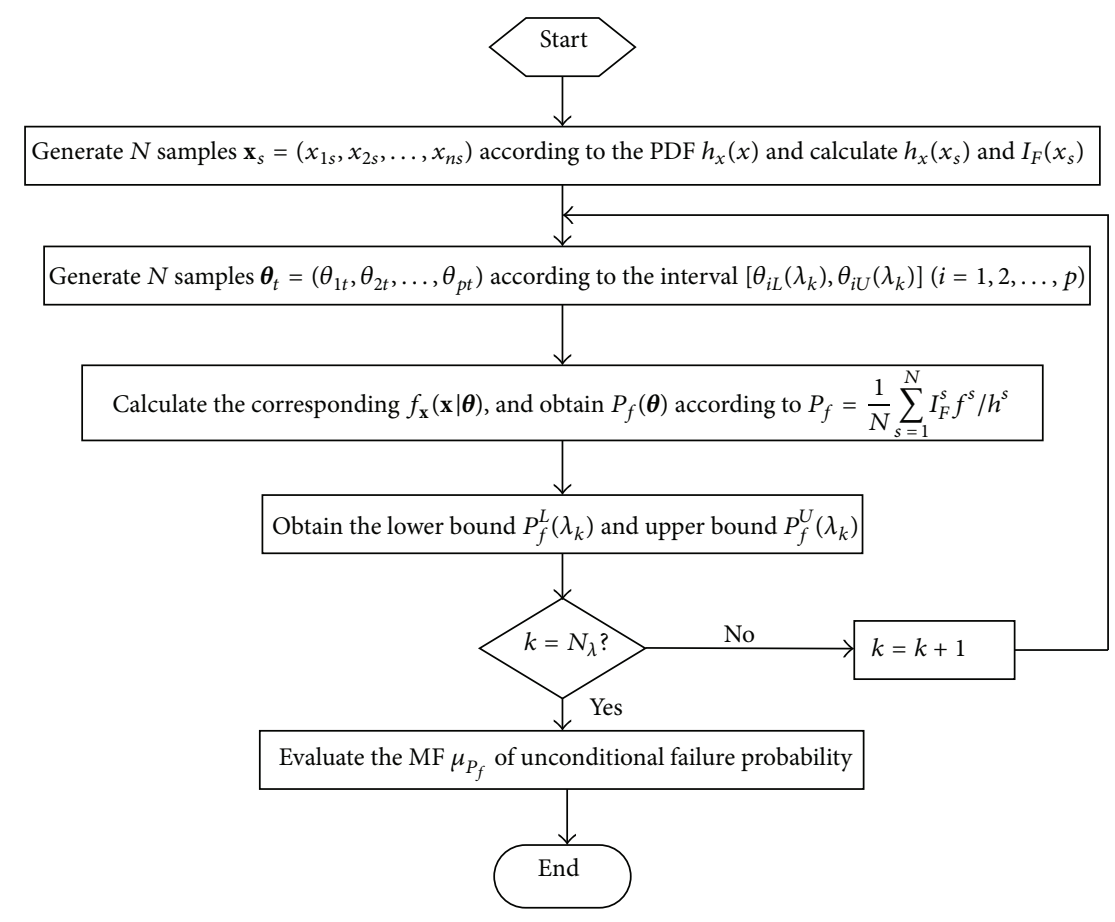

(a) SLS for evaluating MF of unconditional failure probability

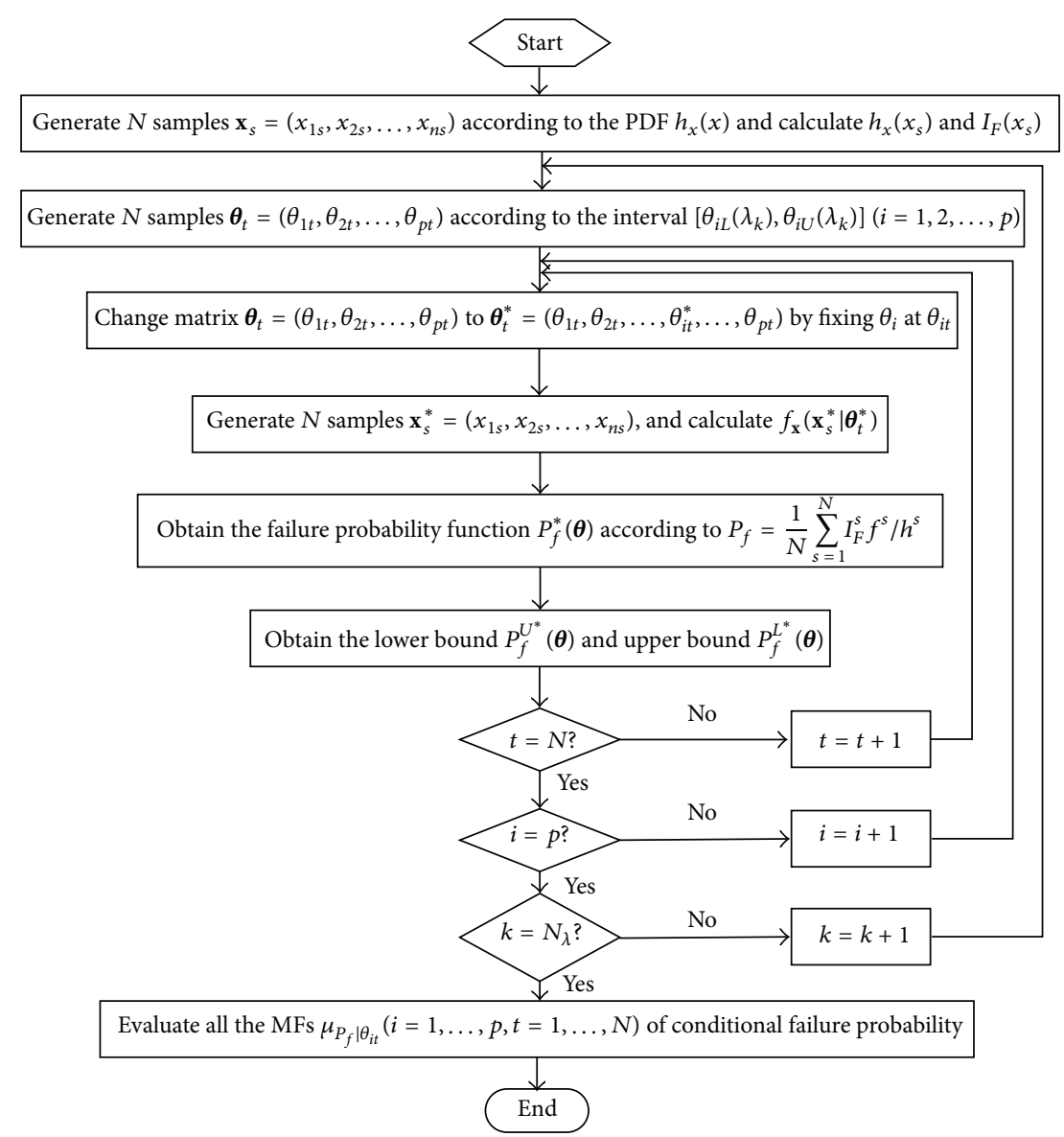

(b) SLS for evaluating MFs of conditional failure probability

FIGURE 3: Flow chart of SLS for evaluating the MFs of unconditional and conditional failure probability. 


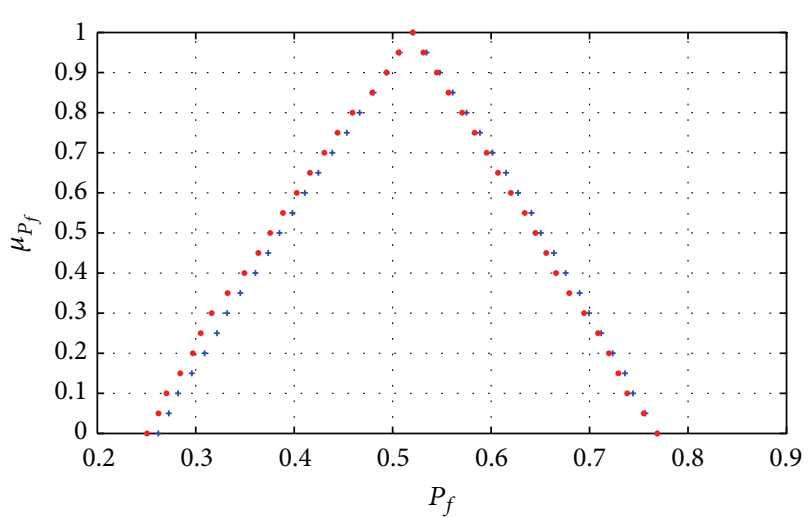

+ MCS

* SLS

Figure 4: MFs of failure probability for Example 1, Case 1.

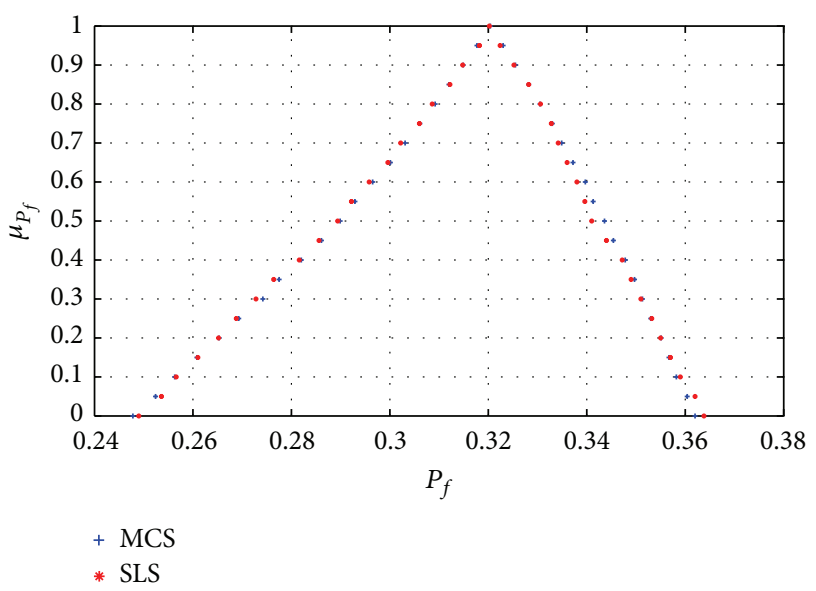

Figure 5: MFs of failure probability for Example 1, Case 2.

Example 2. The response function $g=\sin x_{1}+7 \sin ^{2} x_{2}+$ $0.1 x_{3}^{4} \sin x_{1}$ includes three variables, where $x_{i} \sim N\left(0, \sigma_{i}\right)$. And the MFs of distribution parameters $\sigma_{i}$ are given as

$$
\mu_{\sigma_{i}}\left(\sigma_{i}\right)= \begin{cases}0 & \sigma_{i} \leq 0.3 \pi \\ \frac{5}{\pi \sigma_{i}}-1.5 & 0.3 \pi \leq \sigma_{i} \leq 0.5 \pi \\ 1 & 0.5 \pi \leq \sigma_{i} \leq 0.7 \pi \\ 8-\frac{10 \sigma_{i}}{\pi} & 0.7 \pi \leq \sigma_{i} \leq 0.8 \pi \\ 0 & \sigma_{i} \geq 0.8 \pi\end{cases}
$$

The MFs of failure probability are drawn in Figure 8, and the results of sensitivity indices calculated by MCS and SLS combing with optimization techniques are listed in Table 3.

Results of sensitivity indices in Table 3 indicate that the fuzzy-valued parameter $\sigma_{3}$ makes more contribution to failure probability of the structural model than the other parameters. And the results evaluated by SLS are in good agreement with those evaluated by MCS.

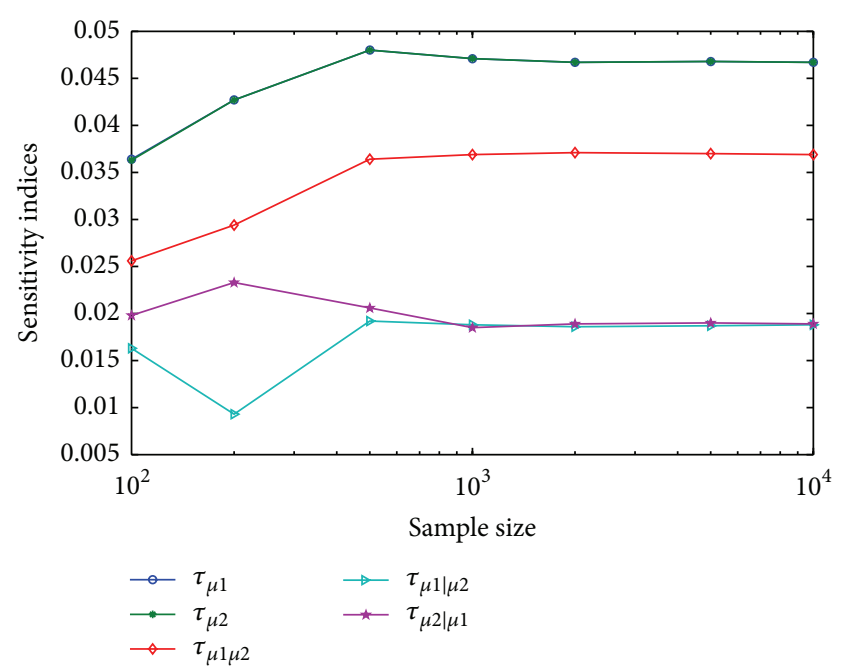

FIGURE 6: Convergence plot of MCS for Example 1, Case 1.

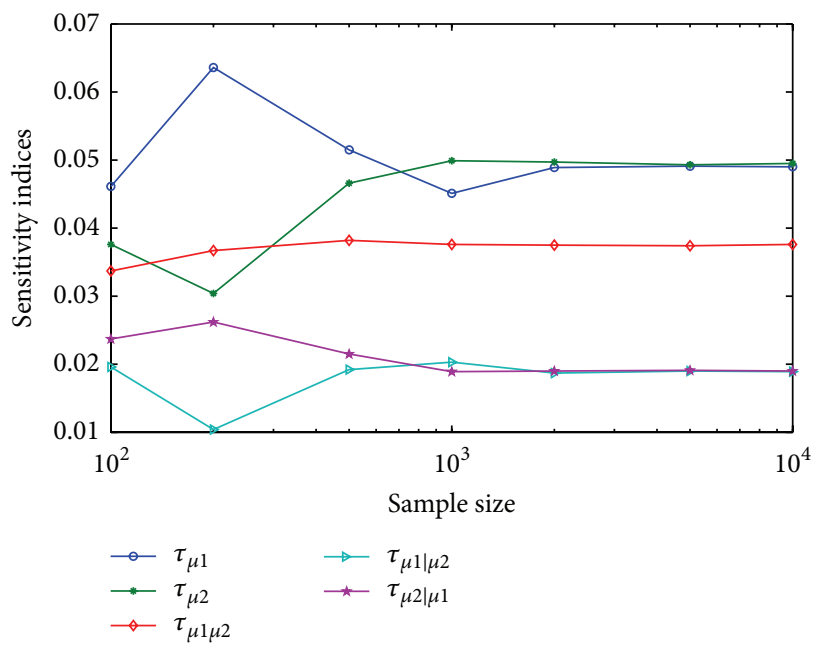

Figure 7: Convergence plot of SLS for Example 1, Case 1.

TABLE 3: Results of global sensitivity indices for Example 2.

\begin{tabular}{lcc}
\hline & $\mathrm{MC}$ & $\mathrm{SL}$ \\
\hline$\left\{\Gamma_{\sigma_{1}}, \Gamma_{\sigma_{2}}, \Gamma_{\sigma_{3}}\right\}$ & $\{0.0230,0.0207,0.0278\}$ & $\{0.0228,0.0201,0.0291\}$ \\
$\left\{\Gamma_{\sigma_{1} \sigma_{2}}, \Gamma_{\sigma_{1} \sigma_{3}}, \Gamma_{\sigma_{2} \sigma_{3}}\right\}$ & $\{0.0213,0.0318,0.0308\}$ & $\{0.0221,0.0336,0.0324\}$ \\
$\left\{\Gamma_{\sigma_{1} \mid \sigma_{2}}, \Gamma_{\sigma_{1} \mid \sigma_{3}}\right\}$ & $\{0.0191,0.0342\}$ & $\{0.0195,0.0361\}$ \\
$\left\{\Gamma_{\sigma_{2} \mid \sigma_{1}}, \Gamma_{\sigma_{2} \mid \sigma_{3}}\right\}$ & $\{0.0169,0.0294\}$ & $\{0.0187,0.0302\}$ \\
$\left\{\Gamma_{\sigma_{3} \mid \sigma_{1}}, \Gamma_{\sigma_{3} \mid \sigma_{2}}\right\}$ & $\{0.0371,0.0376\}$ & $\{0.0369,0.0381\}$ \\
\hline
\end{tabular}

Example 3. A roof truss is shown in Figure 9, and the top boom and the compression bars are reinforced by concrete, and the bottom boom and the tension bars are steel. Assume the uniformly distributed load $q$ is applied on the roof truss, and the uniformly distributed load can be transformed into the nodal load $P=q l / 4$. Taking the safety and applicability into account, the perpendicular deflection $\Delta_{C}$ of the peak of structure node $C$ not exceeding $2.8 \mathrm{~cm}$ is taken as the constraint condition, and the performance response function 
TABLE 4: Distribution parameters of the input variables of roof truss.

\begin{tabular}{lcccccc}
\hline Random variables & $q(\mathrm{~N} / \mathrm{m})$ & $l(\mathrm{~m})$ & $A_{S}\left(\mathrm{~m}^{2}\right)$ & $A_{C}\left(\mathrm{~m}^{2}\right)$ & $E_{S}\left(\mathrm{~N} / \mathrm{m}^{2}\right)$ & $E_{C}\left(\mathrm{~N} / \mathrm{m}^{2}\right)$ \\
\hline Mean $\mu_{x}$ & 20000 & 12 & $9.82 \times 10^{-4}$ & 0.04 & $1 \times 10^{11}$ & $2 \times 10^{10}$ \\
Standard deviation $\sigma_{x}$ & $\sigma_{q}$ & $\sigma_{l}$ & $\sigma_{A_{s}}$ & $\sigma_{A_{c}}$ & $\sigma_{E_{s}}$ & $\sigma_{E_{c}}$ \\
\hline
\end{tabular}

TABLE 5: Coefficients for membership function of $\sigma_{x}$.

\begin{tabular}{lcccccc}
\hline Standard deviation $\sigma_{x}$ & $\sigma_{q}$ & $\sigma_{l}$ & $\sigma_{A_{s}}$ & $\sigma_{A_{c}}$ & $\sigma_{E_{s}}$ \\
\hline Coefficient $a$ & 1400 & 0.12 & $5.892 \times 10^{-5}$ & 0.0048 & $6 \times 10^{9}$ & $1.2 \times 10^{9}$ \\
Coefficient $b$ & 700 & 0.006 & $2.95 \times 10^{-6}$ & 0.0002 & $3 \times 10^{8}$ & $6 \times 10^{7}$ \\
\hline
\end{tabular}

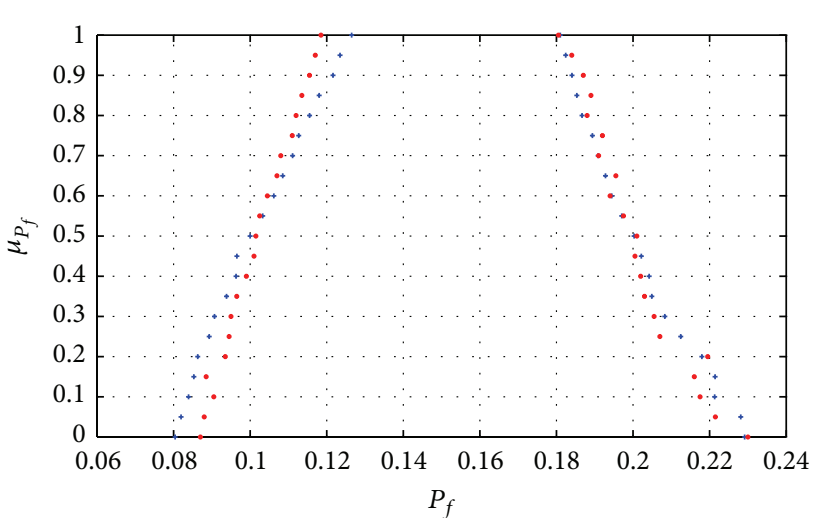

+ MCS

* SLS

Figure 8: MFs of failure probability for Example 2.

can be constructed by $g(x)=0.028-\Delta_{C}$, where $\Delta_{C}$ is the function of the basic random variables and $\Delta_{C}=$ $\left(q l^{2} / 2\right)\left(3.81 / A_{C} E_{C}+1.13 / A_{S} E_{S}\right)$, and $A_{C}, A_{S}, E_{C}, E_{S}$, and $l$, respectively, are sectional area, elastic modulus, length of the concrete, and steel bars; the distribution parameters of these independent normal basic random variables are listed in Table 4. It is assumed that the standard deviation of the variables is fuzzy parameters with membership function $\mu_{\sigma_{i}}\left(\sigma_{i}\right)=\exp \left(-(x-a)^{2} / 2 b^{2}\right)$. And the coefficients are listed in Table 5.

The MFs of failure probability are drawn in Figure 10, and the results of sensitivity indices calculated by MCS and SLS combing with optimization techniques are listed in Table 6.

Results of sensitivity indices in Table 6 show that the rank of fuzzy valued distribution parameter is $\sigma_{A_{s}}>\sigma_{E_{s}}>$ $\sigma_{A_{c}}>\sigma_{E_{c}}>\sigma_{l}>\sigma_{q}$, among which $\sigma_{q}$ has little effect on the failure probability of the fuzzy structural model, and the effect of $\sigma_{q}$ with other parameters is the smallest among all the $\Gamma_{\theta_{i} \theta_{i}}$. In addition, the sensitivity indices can be estimated by the SLS guaranteeing acceptable accuracy with only one set of samples, which can greatly improve the computational efficiency. As testified by the results of Examples 1-3, properties of the sensitivity indices are obviously satisfied.

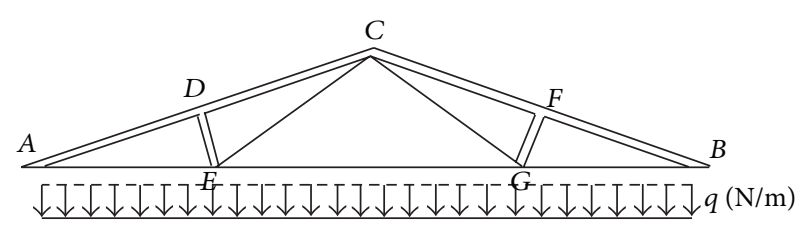

(a)

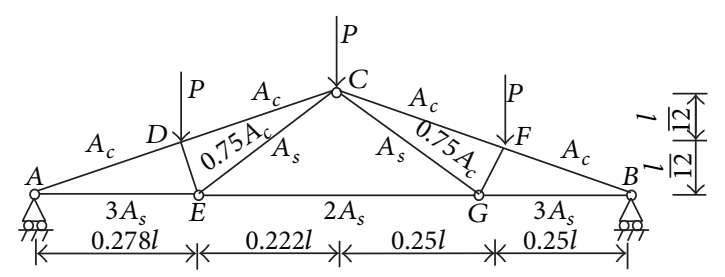

(b)

FIGURE 9: Schematic diagram of a roof truss.

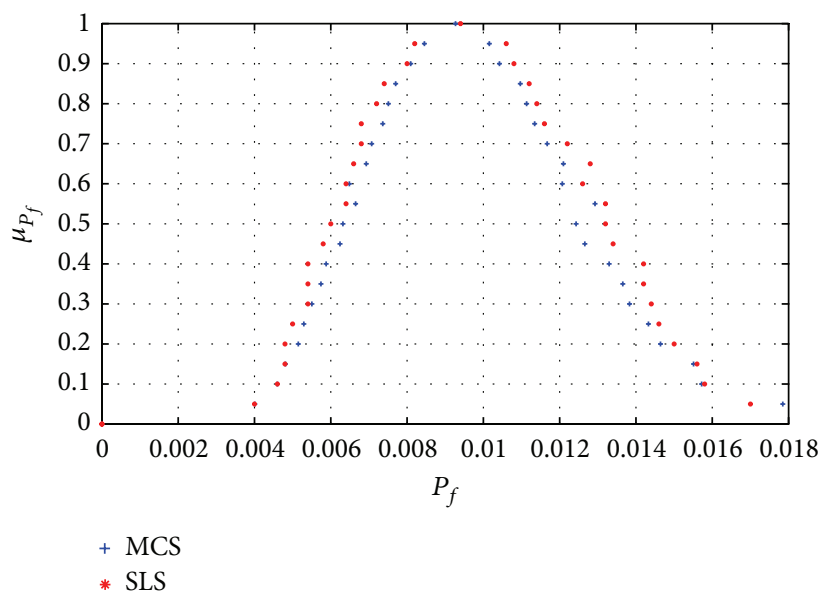

FIGURE 10: MFs of failure probability for Example 3.

\section{Conclusion}

A global sensitivity index of fuzzy-valued distribution parameter on the fuzzy failure probability is defined in this work. The key to calculate the sensitivity index is to evaluate the MFs of failure probability. For the problem that the computational cost of MCS is rather expensive, a SLS is introduced to estimate the proposed sensitivity indices, 
TABLE 6: Results of global sensitivity indices for Example 3.

\begin{tabular}{|c|c|c|}
\hline & MCS & SLS \\
\hline$\left\{\Gamma_{\sigma_{q}}, \Gamma_{\sigma_{l}}, \Gamma_{\sigma_{A_{s}}}, \Gamma_{\sigma_{A_{c}}}, \Gamma_{\sigma_{E_{s}}}, \Gamma_{\sigma_{E_{c}}}\right\}$ & $\begin{array}{c}10^{-3} \times \\
\{0.2553,0.4535,0.8478,0.7000,0.7010,0.4694\}\end{array}$ & $\begin{array}{c}10^{-3} \times \\
\{0.2385,0.4412,0.8324,0.7132,0.6829,0.4367\}\end{array}$ \\
\hline$\left\{\Gamma_{\sigma_{q} \sigma_{l}}, \Gamma_{\sigma_{q} \sigma_{A_{s}}}, \Gamma_{\sigma_{q} \sigma_{A_{c}}}, \Gamma_{\sigma_{q} \sigma_{E_{s}}}, \Gamma_{\sigma_{q} \sigma_{E_{c}}}\right\}$ & $\{0.0009,0.0010,0.0011,0.0009,0.0009\}$ & $\{0.0007,0.0008,0.0009,0.0007,0.0007\}$ \\
\hline$\left\{\Gamma_{\sigma_{l} \sigma_{A_{s}}}, \Gamma_{\sigma_{l} \sigma_{A_{c}}}, \Gamma_{\sigma_{l} \sigma_{E_{s}}}, \Gamma_{\sigma_{l} \sigma_{E_{c}}}\right\}$ & $\{0.0027,0.0032,0.0025,0.0024\}$ & $\{0.0023,0.0028,0.0022,0.0021\}$ \\
\hline$\left\{\Gamma_{\sigma_{A_{s}} \sigma_{A_{c}}}, \Gamma_{\sigma_{A_{s}} \sigma_{E_{s}}}, \Gamma_{\sigma_{A_{s}} \sigma_{E_{c}}}\right\}$ & $\{0.0035,0.0029,0.0027\}$ & $\{0.0028,0.0025,0.0023\}$ \\
\hline$\left\{\Gamma_{\sigma_{A_{c}} \sigma_{E_{s}}}, \Gamma_{\sigma_{A_{c}} \sigma_{E_{c}}}\right\}$ & $\{0.0033,0.0032\}$ & $\{0.0027,0.0026\}$ \\
\hline$\left\{\Gamma_{\sigma_{E_{s}} \sigma_{E_{c}}}\right\}$ & $\{0.0024\}$ & $\{0.0021\}$ \\
\hline$\left\{\Gamma_{\sigma_{q} \mid \sigma_{l}}, \Gamma_{\sigma_{q} \mid \sigma_{A_{s}}}, \Gamma_{\sigma_{q} \mid \sigma_{A_{c}}}, \Gamma_{\sigma_{q} \mid \sigma_{E_{s}}}, \Gamma_{\sigma_{q} \mid \sigma_{E_{c}}}\right\}$ & $\{0.0024,0.0023,0.0029,0.0024,0.0024\}$ & $\{0.0021,0.0020,0.0027,0.0021,0.0021\}$ \\
\hline$\left\{\Gamma_{\sigma_{l} \mid \sigma_{q}}, \Gamma_{\sigma_{l} \mid \sigma_{A_{s}}}, \Gamma_{\sigma_{l} \mid \sigma_{A_{c}}}, \Gamma_{\sigma_{l} \mid \sigma_{E_{s}}}, \Gamma_{\sigma_{l} \mid \sigma_{E_{c}}}\right\}$ & $\{0.0023,0.0070,0.0084,0.0069,0.0069\}$ & $\{0.0020,0.0064,0.0078,0.0064,0.0064\}$ \\
\hline$\left\{\Gamma_{\sigma_{A_{s}} \mid \sigma_{q}}, \Gamma_{\sigma_{A_{s}} \mid \sigma_{l}}, \Gamma_{\sigma_{A_{s}} \mid \sigma_{A_{c}}}, \Gamma_{\sigma_{A_{s}} \mid \sigma_{E_{s}}}, \Gamma_{\sigma_{A_{s}} \mid \sigma_{E_{c}}}\right\}$ & $\{0.0023,0.0072,0.0087,0.0073,0.0072\}$ & $\{0.0020,0.0066,0.0080,0.0067,0.0067\}$ \\
\hline$\left\{\Gamma_{\sigma_{A_{c}} \mid \sigma_{q}}, \Gamma_{\sigma_{A_{c}} \mid \sigma_{l}}, \Gamma_{\sigma_{A_{c}} \mid \sigma_{A_{s}}}, \Gamma_{\sigma_{A_{c}} \mid \sigma_{E_{s}}}, \Gamma_{\sigma_{A_{c}} \mid \sigma_{E_{c}}}\right\}$ & $\{0.0028,0.0085,0.0085,0.0085,0.0085\}$ & $\{0.0024,0.0080,0.0080,0.0080,0.0080\}$ \\
\hline$\left\{\Gamma_{\sigma_{E_{s}} \mid \sigma_{q}}, \Gamma_{\sigma_{E_{s}} \mid \sigma_{l}}, \Gamma_{\sigma_{E_{s}} \mid \sigma_{A_{s}}}, \Gamma_{\sigma_{E_{s}} \mid \sigma_{A_{c}}}, \Gamma_{\sigma_{E_{s}} \mid \sigma_{E_{c}}}\right\}$ & $\{0.0024,0.0072,0.0073,0.0087,0.0070\}$ & $\{0.0021,0.0066,0.0066,0.0080,0.0064\}$ \\
\hline$\left\{\Gamma_{\sigma_{E_{c}} \mid \sigma_{q}}, \Gamma_{\sigma_{E_{c}} \mid \sigma_{l}}, \Gamma_{\sigma_{E_{c}} \mid \sigma_{A_{s}}}, \Gamma_{\sigma_{E_{c}} \mid \sigma_{A_{c}}}, \Gamma_{\sigma_{E_{c}} \mid \sigma_{E_{s}}}\right\}$ & $\{0.0023,0.0070,0.0071,0.0085,0.0070\}$ & $\{0.0020,0.0064,0.0064,0.0067,0.0064\}$ \\
\hline
\end{tabular}

which can greatly improve the computational efficiency considerably with acceptable accuracy. Applications of the sensitivity indices proposed in this paper to the numerical and engineering examples show that fuzzy sensitivity indices can provide useful information about the sequence of the distribution parameters on the fuzzy uncertainty of failure probability, and the proposed sensitivity indices can identify the relative important fuzzy distribution parameters of the structural model, thus providing useful information for engineers in the process of reliability design and optimization.

\section{Conflict of Interests}

The authors declare that there is no conflict of interests regarding the publication of this paper.

\section{Acknowledgments}

This work was supported by the National Natural Science Foundation of China (Grant nos. NSFC 51175425), the Aviation Foundation (Grant nos. 2011ZA53015), and the Special Research Fund for the Doctoral Program of Higher Education of China (Grant nos. 20116102110003).

\section{References}

[1] A. Der Kiureghian and O. Ditlevsen, "Aleatory or epistemic? Does it matter?" Structural Safety, vol. 31, no. 2, pp. 105-112, 2009.

[2] J. Guo and X. Du, "Sensitivity analysis with mixture of epistemic and aleatory uncertainties," AIAA Journal, vol. 45, no. 9, pp. 2337-2349, 2007.

[3] J. C. Helton, "Alternative representations of epistemic uncertainty," Reliability Engineering and System Safety, vol. 85, no. 1-3, pp. 1-10, 2004.

[4] S. S. Rao and L. Berke, "Analysis of uncertain structural systems using interval analysis," AIAA Journal, vol. 35, no. 4, pp. 727735, 1997.
[5] R. Moore and W. Lodwick, "Interval analysis and fuzzy set theory," Fuzzy Sets and Systems, vol. 135, no. 1, pp. 5-9, 2003.

[6] Z. Qiu, "Convex models and interval analysis method to predict the effect of uncertain-but-bounded parameters on the buckling of composite structures," Computer Methods in Applied Mechanics and Engineering, vol. 194, no. 18-20, pp. 2175-2189, 2005.

[7] J. Kohlas, "Allocation of arguments and evidence theory," Theoretical Computer Science, vol. 171, no. 1-2, pp. 221-246, 1997.

[8] J. C. Helton, J. D. Johnson, and W. L. Oberkampf, "An exploration of alternative approaches to the representation of uncertainty in model predictions," Reliability Engineering and System Safety, vol. 85, no. 1-3, pp. 39-71, 2004.

[9] L. A. Zadeh, "Fuzzy sets," Information and Computation, vol. 8, no. 3, pp. 338-353, 1965.

[10] J. C. Helton and F. J. Davis, "Latin hypercube sampling and the propagation of uncertainty in analyses of complex systems," Reliability Engineering \& System Safety, vol. 81, no. 1, pp. 23-69, 2003.

[11] J. C. Helton and F. J. Davis, "Sampling-based methods," in Sensitivity Analysis, A. Saltelli, K. Chan, and E. M. Scott, Eds., pp. 101-153, John Wiley \& Sons, New York, NY, USA, 2000.

[12] A. Saltelli and J. Marivoet, "Non-parametric statistics in sensitivity analysis for model output: a comparison of selected techniques," Reliability Engineering and System Safety, vol. 28, no. 2, pp. 229-253, 1990.

[13] I. M. Sobol, "Sensitivity analysis for non-linear mathematical models," Mathematical and Computer Modelling, vol. 1, pp. 407414,1993 , English translation of Russian original paper.

[14] I. M. Sobol/, "Global sensitivity indices for nonlinear mathematical models and their Monte Carlo estimates," Mathematics and Computers in Simulation, vol. 55, no. 1-3, pp. 271-280, 2001.

[15] R. L. Iman and S. C. Hora, "A robust measure of uncertainty importance for use in fault tree system analysis," Risk Analysis, vol. 10, no. 3, pp. 401-406, 1990.

[16] E. Castillo, R. Mínguez, and C. Castillo, "Sensitivity analysis in optimization and reliability problems," Reliability Engineering \& System Safety, vol. 93, no. 12, pp. 1788-1800, 2008. 
[17] M. H. Chun, S. J. Han, and N. I. Tak, "An uncertainty importance measure using a distance metric for the change in a cumulative distribution function," Reliability Engineering and System Safety, vol. 70, no. 3, pp. 313-321, 2000.

[18] H. Liu, W. Chen, and A. Sudjianto, "Relative entropy based method for probabilistic sensitivity analysis in engineering design," Journal of Mechanical Design, vol. 128, no. 2, pp. 326336, 2006.

[19] E. Borgonovo, "A new uncertainty importance measure," Reliability Engineering \& System Safety, vol. 92, no. 6, pp. 771-784, 2007.

[20] W. Castaings, E. Borgonovo, M. D. Morris, and S. Tarantola, "Sampling strategies in density-based sensitivity analysis," Environmental Modelling and Software, vol. 38, pp. 13-26, 2012.

[21] E. Plischke, E. Borgonovo, and C. L. Smith, "Global sensitivity measures from given data," European Journal of Operational Research, vol. 226, no. 3, pp. 536-550, 2013.

[22] X. P. Luo, Z. Z. Lu, and X. A. Xu, "A fast computational method for moment-independent uncertainty importance measure," Computer Physics Communications, vol. 185, no. 1, pp. 19-27, 2014.

[23] E. Borgonovo, W. Castaings, and S. Tarantola, "Model emulation and moment-independent sensitivity analysis: an application to environmental modelling," Environmental Modelling and Software, vol. 34, pp. 105-115, 2012.

[24] B. Anderson, E. Borgonovo, M. Galeotti, and R. Roson, "Uncertainty in climate change modeling: can global sensitivity analysis be of help?" Risk Analysis, vol. 34, no. 2, pp. 271-293, 2014.

[25] P. Wei, Z. Lu, W. Hao, J. Feng, and B. Wang, "Efficient sampling methods for global reliability sensitivity analysis," Computer Physics Communications, vol. 183, no. 8, pp. 1728-1743, 2012.

[26] E. Borgonovo, W. Castaings, and S. Tarantola, "Moment independent importance measures: new results and analytical test cases," Risk Analysis, vol. 31, no. 3, pp. 404-428, 2011.

[27] L. A. Zadeh, "Fuzzy sets as a basis for a theory of possibility," Fuzzy Sets and Systems, vol. 1, no. 1, pp. 3-28, 1978.

[28] V. Braibant, A. Oudshoorn, C. Boyer, and F. Delcroix, "Nondeterministic "possibilistic" approaches for structural analysis and optimal design," AIAA journal, vol. 37, no. 10, pp. 1298-1303, 1999.

[29] M. Hanss and A. Klimke, "On the reliability of the influence measure in the transformation method of fuzzy arithmetic," Fuzzy Sets and Systems, vol. 143, no. 3, pp. 371-390, 2004.

[30] U. Gauger, S. Turrin, M. Hanss, and L. Gaul, "A new uncertainty analysis for the transformation method," Fuzzy Sets and Systems, vol. 159, no. 11, pp. 1273-1291, 2008.

[31] O. Giannini and M. Hanss, "An interdependency index for the outputs of uncertain systems," Fuzzy Sets and Systems, vol. 159, no. 11, pp. 1292-1308, 2008.

[32] D. Dubois and H. Prade, Possibility Theory, Plenum Press, New York, NY, USA, 1988.

[33] S. F. Song, Z. Z. Lu, and L. J. Cui, "A generalized Borgonovo's importance measure for fuzzy input uncertainty," Fuzzy Sets and Systems, vol. 189, pp. 53-62, 2012.

[34] L. Y. Li, Z. Z. Lu, and S. F. Song, "Saddlepoint approximation based line sampling method for uncertainty propagation in fuzzy and random reliability analysis," Science China Technological Sciences, vol. 53, no. 8, pp. 2252-2260, 2010.

[35] T. Zhangchun, L. Zhenzhou, P. Wang, and Z. Feng, "An entropybased global sensitivity analysis for the structures with both fuzzy variables and random variables," Proceedings of the Institution of Mechanical Engineers, Part C: Journal of Mechanical Engineering Science, vol. 227, no. 2, pp. 195-212, 2013.

[36] L. J. Cui, Z. Z. Lu, and P. Wang, "Reliability sensitivity with mixture of random and fuzzy variables," in Proceedings of the 2011 International Conference on Quality, Reliability, Risk, Maintenance and Safety Engineering, vol. 887-892, Xian, China, June 2011.

[37] S. Song, Z. Lu, W. Li, and L. Cui, “The uncertainty importance measures of the structural system in view of mixed uncertain variables," Fuzzy Sets and Systems, vol. 243, pp. 25-35, 2014.

[38] P. Wang, Z. Lu, and Z. Tang, "A derivative based sensitivity measure of failure probability in the presence of epistemic and aleatory uncertainties," Computers \& Mathematics with Applications, vol. 65, no. 1, pp. 89-101, 2013.

[39] L. Y. Li, Z. Z. Lu, and W. Li, "State dependent parameter method for importance analysis in the presence of epistemic and aleatory uncertainties," Science China Technological Sciences, vol. 55, no. 6, pp. 1608-1617, 2012.

[40] P. F. Wei, Z. Z. Lu, and J. W. Song, "Extended monte carlo simulation for parametric global sensitivity analysis optimization," AIAA Journal, vol. 52, no. 4, pp. 867-878, 2014.

[41] L. A. Zadeh, "Probability measures of fuzzy events," Journal of Mathematical Analysis and Applications, vol. 23, pp. 421-427, 1968.

[42] D. Dubois and H. Prade, "The three semantics of fuzzy sets," Fuzzy Sets and Systems, vol. 90, no. 2, pp. 141-150, 1997.

[43] D. Dubois and H. Prade, Possibility Theory: An Approach to Computerized Processing of Uncertainty, Plenum Press, New York, NY. USA, 1988.

[44] C. H. Liu and Q. Chen, "Analysis of structural general reliability of the loading and strength based on information entropy," Mechanical Science and Technology, vol. 22, no. 3, pp. 444-446, 2003.

[45] E. Borgonovo, S. Tarantola, E. Plischke, and M. D. Morris, "Transformation and invariance in the sensitivity analysis of computer experiment," Journal of the Royal Statistical Society B, 2014.

[46] M. Baucells and E. Borgonovo, "Invariant probabilistic sensitivity analysis," Management Science, vol. 59, no. 11, pp. 2536-2549, 2013. 


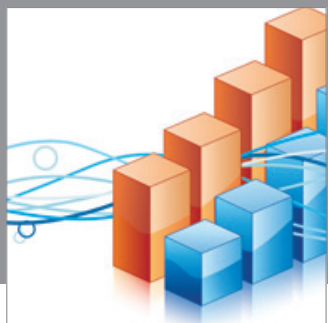

Advances in

Operations Research

mansans

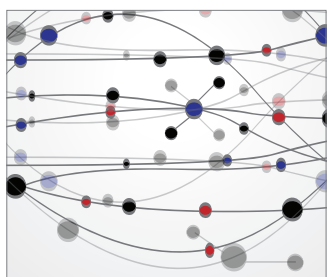

The Scientific World Journal
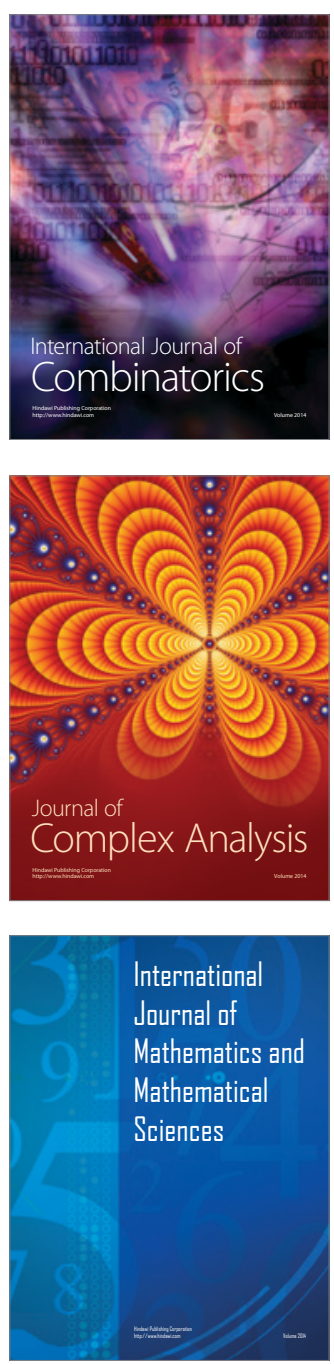
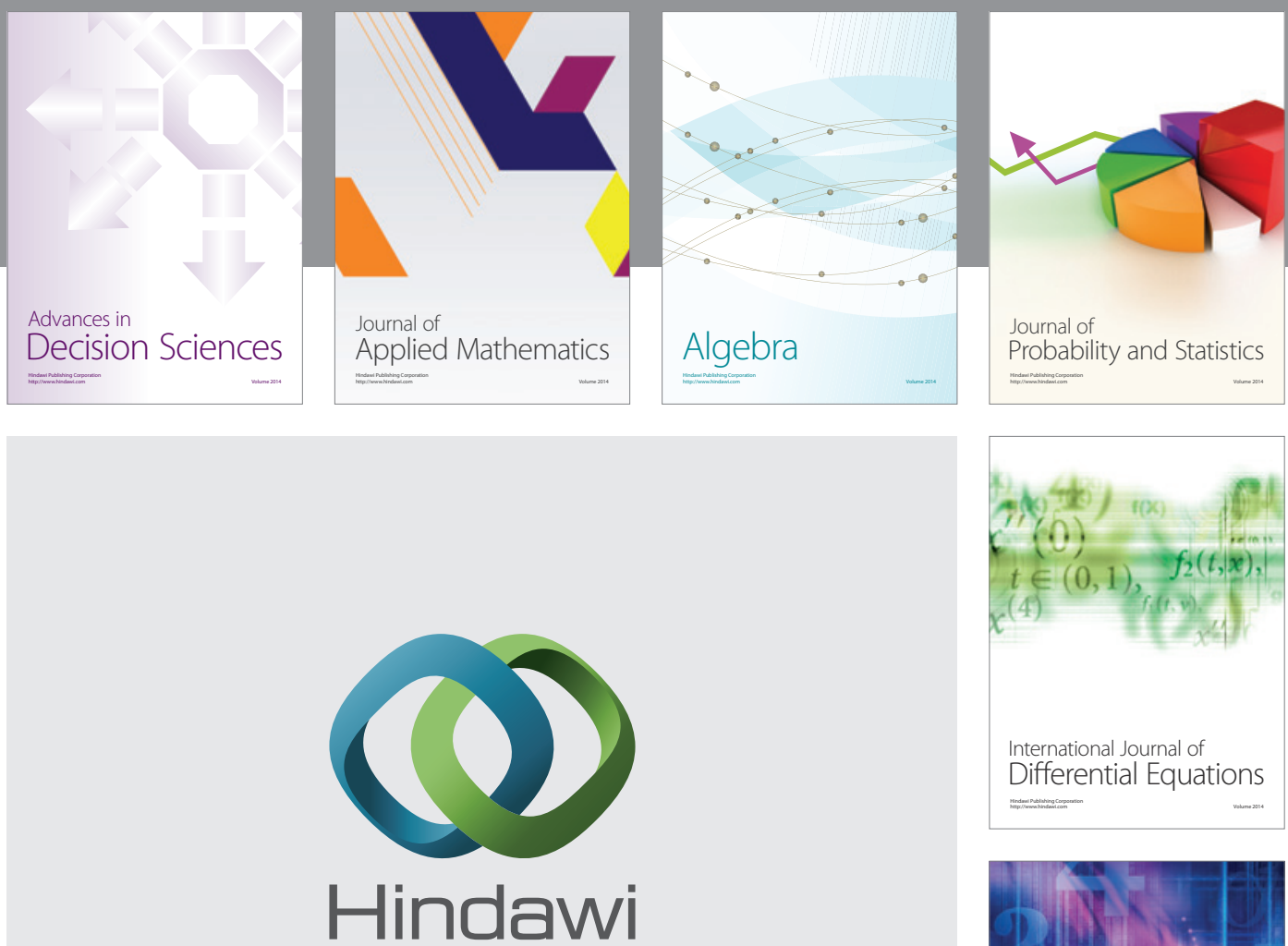

Submit your manuscripts at http://www.hindawi.com
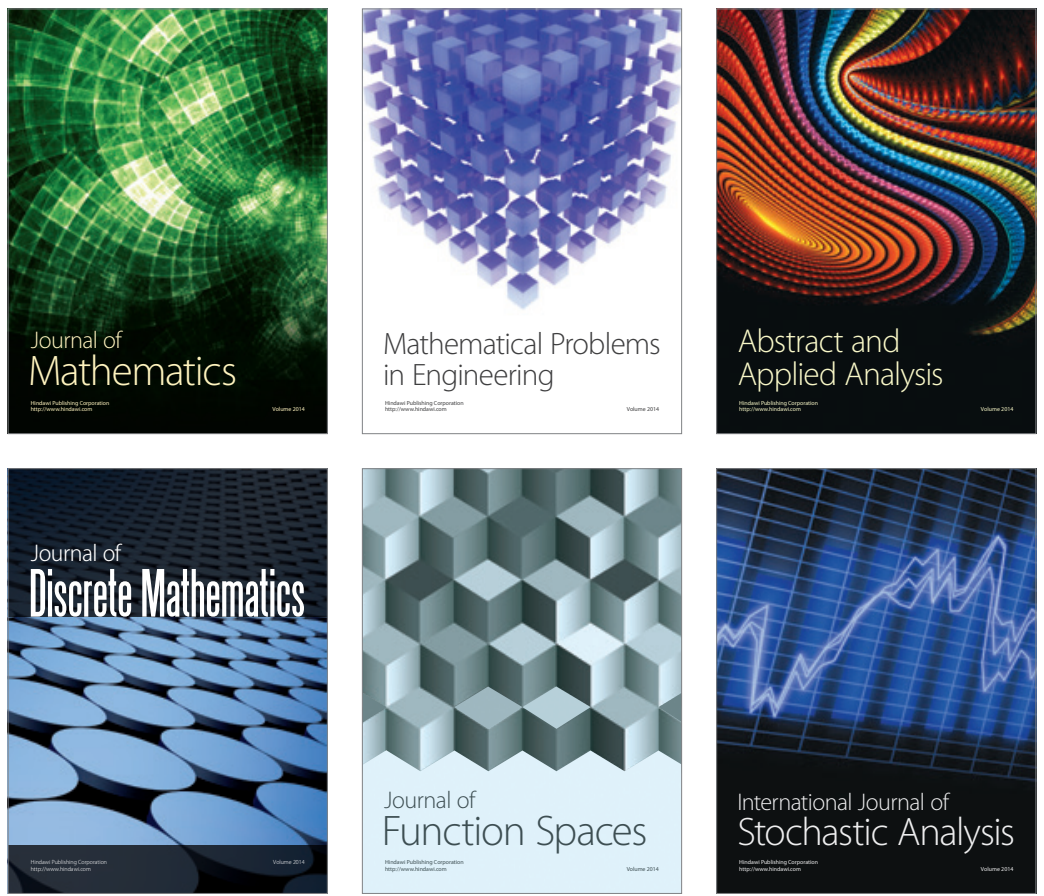

Journal of

Function Spaces

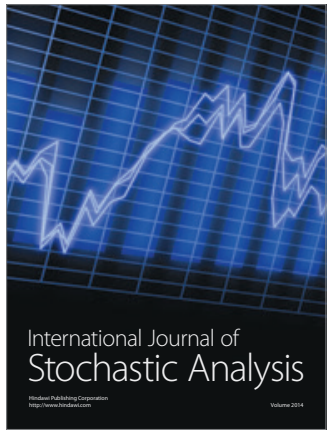

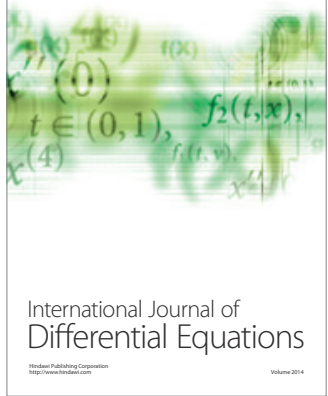
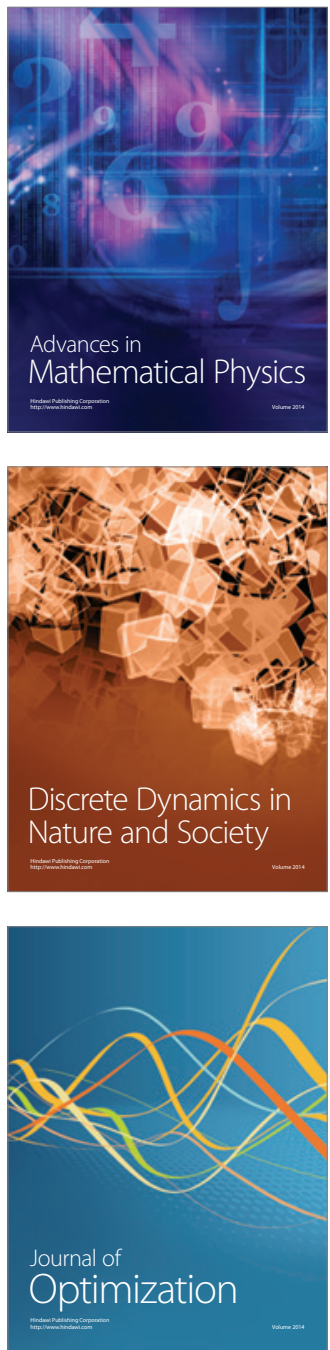